\title{
Identification of Multi-sinusoidal Signals with Direct Frequency Estimation: An Adaptive Observer Approach *
}

\author{
Gilberto Pin ${ }^{\mathrm{a}}$, Yang Wang ${ }^{\mathrm{b}}$, Boli Chen ${ }^{\mathrm{b}}$, Thomas Parisini ${ }^{\mathrm{b}, \mathrm{c}, \mathrm{d}}$ \\ ${ }^{\text {a }}$ Electrolux Italia S.p.A, Italy \\ ${ }^{\mathrm{b}}$ Dept. of Electrical and Electronic Engineering, Imperial College London, UK \\ ${ }^{\mathrm{c}}$ Dept. of Engineering and Architecture, University of Trieste, Italy \\ ${ }^{\mathrm{d}}$ KIOS Research and Innovation Centre of Excellence, University of Cyprus
}

\begin{abstract}
This paper addresses the problem of estimating the frequencies, amplitudes and phases of the $n$ sinusoidal components of a possibly biased multi-sinusoidal signal. The proposed adaptive observer allows the direct adaptation of the frequency estimates with a relatively low dynamic order $3 n+1$ ( $3 n$ for an unbiased signal). The stability analysis proves the global exponential convergence of the estimation error and the robustness to additive norm-bounded measurement perturbations.
\end{abstract}

Key words: Sinusoidal signals; Frequency estimation; Adaptive systems; Estimation algorithms.

\section{Introduction}

The problem of determining the unknown amplitudes, frequencies and phases (AFP) of the components of a multi-sinusoidal signal represents a fundamental challenge in many engineering fields, including active noise and vibration control, periodic disturbance rejection, power quality monitoring, etc. (see, for instance the recent Special Issue [3] and the references cited therein).

The Fast Fourier Transform (FFT) is the most used signal processing technique when dealing with stationary signals, but the accuracy of FFT is known to degrade in the presence of time-varying frequency. Moreover, since it operates over batches of data samples, it requires quite large memory storage resources specially when high resolution is required. These weaknesses motivated the research on alternative AFP methods. In particular, frequency trackers based on Kalman and Extended Kalman Filters (e.g [27], [2]), adaptive-notch-filtering method

\footnotetext{
‡ The dissemination of this work is being supported by the European Union's Horizon 2020 research and innovation programme under grant agreement No 739551 (KIOS CoE).

Email addresses: gilbertopin@alice.it (Gilberto Pin), yang.wang13@imperial.ac.uk (Yang Wang),

boli.chen10@imperial.ac.uk (Boli Chen),

t.parisini@gmail.com (Thomas Parisini).
}

(ANF) (e.g. [16]) and frequency-adaptive Phase-LockedLoop (PLL) (e.g. [19], [30]) represent valid alternatives that offer superior transient perfomances. The said AFP methods have found applications in particular in the estimation of power-electrical signals for monitoring tasks and power quality assessment. To deal with nonzeromean sinusoidal signals arising in practical applications, several modifications to conventional ANF an PLL have been proposed in the recent years, such as Enhanced PLL (EPLL), Quadratic PLL (QPLL), frequency lock loop (FLL) and the second-order generalized integratorbased orthogonal signal generator (OSG-SOGI) (see, for example, [18], [11] and [9] and the references cited therein). Furthermore, higher-order perturbations modeled as time-polynomials (such as drift phenomena) are addressed in [25] and [7] by pre-filtering techniques. With the aim of extending single-tone PLL and ANF structures to the multi-sinusoidal case, combinations of multiple ANFs and PLLs in the form of filter-banks are proposed in [22], [21], [12] and [10]. The main drawback of these approaches is that only local stability proofs are available. Moreover, there exist practical pathological situations in which these estimators fail to converge, such as in the case of sudden frequency variations or in the case of multi-sinusoids with narrow frequency separation.

The ability to provide global or semi-global convergence 
guarantees in case of multiple sinusoids is a notable feature of another class of AFP methods based on adaptive observer (see [23], [31], [14], [28], [15], [28] and [5]). The first algorithm of this class is presented in [20], in which a $5 n^{\text {th }}$-order identifier is designed to cope with $n$ frequencies and the unknown offset, while in [15], a $(5 n+1)^{\text {th }}$ order scheme is presented for the extraction of all the $3 n$ parameters (full AFP estimation). The dynamic order of the adaptive observers is further reduced in [23], [31] and [14], resulting in estimators of dimension $3 n$ for unbiased signals and $(3 n+1)^{\mathrm{th}}$-order if the bias is considered. In the more recent contributions [4] and [26] the dimension of the adaptive system is further reduced to $3 n^{\text {th }}$ for a biased multi-sinusoidal signal. While dimensionality reduction is an important aspect to decrease the complexity of the adaptive observer, consistent effort has been devoted also to increase the robustness of the algorithm in facing saturated signals or unstructured measurement perturbations. For instance, an hybrid observer is proposed in [5] (see also [6]) to identify the $n$ frequencies of a multi-frequency signal with saturation. Moreover, the new methodology called Dynamic Regressor Extension and Mixing (DREM) proposed in [1] and [29] allows to improve at the same time the noise immunity and the transient performance.

However, the common drawback of all the aforementioned multi-sinusoidal AFP methods methods is that the frequencies of the sinusoidal components are not directly estimated. Indeed, the parameters undergoing adaptation correspond to the coefficients of the characteristic polynomial of the autonomous signal generator system. The parametrization of the AFP problem in terms of the coefficients of the characterstic polynomial is used mainly to recast the identification problem in a convenient linerin-the parameters form. However, the frequencies can be then retrieved only at the cost of performing a further - possibly cumbersome (depending on the dimensionality of the problem) - processing step, which consists in finding the complex roots of the characteristic polynomial. As a consequence, the computational burden may increase significantly in the presence of a large number of sinusoids. In order to alleviate the drawback of the afore-described methods, two novel adaptive-observer algorithms have been recently proposed in [8] and [24], that allow to adapt the frequencies directly without the need of further processing.

Based on the preliminary results presented in [24], this paper deals with a full AFP methodology, characterized by a direct adaptation mechanism for the squares of the frequencies. In contrast with the recently presented adaptive observer with the same notable feature [8], the devised method removes the requirement of state augmentation, reducing the dimensionality of the observer.

The paper is organized as follows: Section 2 introduces the AFP problem in the multi-sinusoidal signal scenario. The adaptive observer-based estimator is proposed in section 3 . Then, the stability analysis is dealt with in Section 4. Finally, in Section 5, simulation are given showing the effectiveness of the proposed algorithm.

\section{Problem Formulation}

Consider the perturbed multi-sinusoidal signal:

$$
y(t)=a_{0}+\sum_{i=1}^{n} a_{i} \sin \left(\phi_{i}+\omega_{i} t\right)+d(t), t \in \mathbb{R}_{\geq 0}
$$

where $a_{0} \in \mathbb{R}_{>0}$ is the unknown constant bias. The amplitudes of the sinusoidal components verify the inequality $a_{i} \geq 0$ and $\phi_{i}$ represents the unknown initial phase of each sinusoid. The frequencies are time-invariant parameters subject to: $\omega_{i}>0, \omega_{i} \neq \omega_{j}$ for $i \neq j$. The term $d(t)$ denotes the measurement disturbance.

The following assumptions are used in this paper.

Assumption 1 The squared-frequencies of the sinusoids are bounded by a known positive constant $\bar{\Omega}$ such that $\omega_{i}^{2}<\bar{\Omega}, \forall i \in\{1,2, \cdots, n\}$.

Assumption 2 The additive measurement perturbation $d(t)$ verifies the following constraints:

$$
|d(t)| \leq \bar{d}_{1}, \quad \dot{d}(t) \mid \leq \bar{d}_{2}, \quad \forall t \in \mathbb{R}_{\geq 0}
$$

where $\bar{d}_{1}$ and $\bar{d}_{2}$ are known positive constants.

Remark 2.1 In practical applications, there exists a variety of perturbations which can be characterised by the conditions proposed in Assumption 2, such as a linear drifts, band-limited white noises and additional (unstructured) sinusoids with finite amplitudes.

The signal $y(t)$ can be thought as generated by the following autonomous multi-harmonic oscillator comprised of $n$ second-order linear harmonic oscillators:

$$
\begin{aligned}
& \dot{z}(t)=\operatorname{diag}\left(G_{1} \cdots G_{n}, 0\right) z(t) \\
& y(t)=C z(t)+d(t)
\end{aligned}
$$

with $z(t) \triangleq\left[z_{1}(t), \cdots, z_{2 n+1}(t)\right] \in \mathbb{R}^{2 n+1}$. The matrices $G_{i}$ and $C$ are given by:

$$
\begin{aligned}
G_{i} & =\left[\begin{array}{cc}
0 & \frac{\omega_{i}^{2}}{r^{2}} \\
-r^{2} & 0
\end{array}\right], \forall i \in\{1, \ldots, n\} \\
C^{\top} & =\left[\begin{array}{llllllll}
1 & 0 & 1 & 0 & \cdots & 1 & 0 & 1
\end{array}\right]^{\top} \in \mathbb{R}^{2 n+1},
\end{aligned}
$$

where $r \in \mathbb{R}_{>0}$ can be chosen arbitrarily. The initial conditions of the multi-oscillator are related to the initial 
phase of the sinusoids in (1) by

$$
\left[\begin{array}{c}
z_{2 i-1}(0) \\
z_{2 i}(0)
\end{array}\right]=a_{i}\left[\begin{array}{c}
\sin \left(\phi_{i}\right) \\
\frac{r^{2} \cos \left(\phi_{i}\right)}{\omega_{i}}
\end{array}\right], \forall i \in\{1, \ldots, n\}
$$

and $z_{2 n+1}(0)=a_{0}$. The model of the signal generator (2) will be now recast in an adaptive observer form. To this end, let us introduce a vector $\Lambda=\left[\begin{array}{llll}\alpha_{1} & \alpha_{2} & \cdots & \alpha_{n}\end{array}\right]$, parametrized by tuning parameters $\alpha_{i} \in \mathbb{R}_{>0}, i=$ $1,2, \cdots, n$ such that $\alpha_{i} \neq \alpha_{j}$ for $i \neq j$, and the constant

$$
\bar{\alpha} \triangleq \max _{i \in\{1,2 \cdots, n\}}\left\{\alpha_{i}\right\}
$$

Moreover, defining $A \triangleq A(\Lambda)=\operatorname{diag}\left(J_{1}, J_{2}, \cdots, J_{n}, 0\right)$ with $J_{i}$ given by

$$
J_{i}=\left[\begin{array}{cc}
0 & r^{2} \alpha_{i} \\
-r^{2} & 0
\end{array}\right], \forall i=1,2 \ldots, n
$$

Then, the multi-oscillator (2) can be rewritten as:

$$
\begin{aligned}
& \dot{z}(t)=A z(t)+\Gamma(z) \Phi(\Omega) \\
& y(t)=C z(t)+d(t)
\end{aligned}
$$

where $\Omega \triangleq\left[\begin{array}{llll}\omega_{1}^{2} & \omega_{2}^{2} & \cdots & \omega_{n}^{2}\end{array}\right]^{\top}$ is a vector containing the spectral parameters, while $\Gamma(z) \in \mathbb{R}^{(2 n+1) \times n}$ is a sparse matrix where the non-zero components are defined as: $\Gamma_{2 i-1, i}=z_{2 i}, \forall i=1,2, \cdots, n$, and $\Phi(\Omega) \in \mathbb{R}^{n}$ is given by

$$
\begin{aligned}
\Phi(\Omega) & =\left[\Phi_{1}(\Omega) \Phi_{2}(\Omega) \cdots \Phi_{n}(\Omega)\right]^{\top}=\frac{\Omega}{r^{2}}-r^{2} \Lambda \\
& =\left[\frac{\omega_{1}^{2}}{r^{2}}-r^{2} \alpha_{1} \frac{\omega_{2}^{2}}{r^{2}}-r^{2} \alpha_{2} \cdots \frac{\omega_{n}^{2}}{r^{2}}-r^{2} \alpha_{n}\right]^{\top}
\end{aligned}
$$

For the sake of brevity, in the sequel we will drop the dependence of $\Phi(\Omega)$ on $\Omega$, thus letting $\Phi=\Phi(\Omega)$. Note that, thanks to the Assumption 1, the vector $\Omega$ belongs to the compact set $\mathcal{W}$ defined as

$$
\mathcal{W} \triangleq\left\{\Omega \in \mathbb{R}^{n}:|\Omega| \leq \sqrt{n} \bar{\Omega}\right\} .
$$

Moreover, the following further assumption on the state of the signal generator (4) is formulated.

Assumption 3 The norm of the state $z(t)$ is bounded by a known finite positive constant $\bar{z} \in \mathbb{R}_{>0}$, such that $|z(t)| \leq \bar{z}, \forall t \in \mathbb{R}_{\geq 0}$.

According to Assumption 3, it holds that $z(t) \in \mathcal{Z}$, where $\mathcal{Z}$ is a compact set defined as $\mathcal{Z} \triangleq\left\{z \in \mathbb{R}^{2 n+1}\right.$ : $\left.|z| \leq r^{2} \bar{z}\right\}$ for any $r \geq 1$.

\section{Adaptive Observer-based Estimation Scheme}

Now, we introduce the structure of the adaptive observer for the joint estimation of $z(t), \Phi$ and $\omega_{i}, i=1, \cdots, n$. In view of (4), let $\hat{\Phi}(t)$ be an estimated counterpart of $\Phi$ (the dynamics of $\hat{\Phi}(t)$ is described later on by $(9)$ ). We first propose an unconstrained mechanism for state estimation

$$
\dot{\hat{z}}_{\text {pre }}(t) \triangleq A \hat{z}(t)+\Gamma(\hat{z}) \hat{\Phi}(t)+L(y(t)-C \hat{z}(t)),
$$

with the observer gain $L$ designed such that the eigenvalues of $A-L C$, denoted by $g_{1} \ldots g_{2 n+1}$, satisfy the constraint: $g_{i} \in \mathbb{R}_{<0}, \forall i \in\{1, \cdots, 2 n+1\}$. Then, with the aim of confining the estimated state $\hat{z}(t)$ to the predefined convex region $\mathcal{Z}$, a projection operator is utilized, which gives rise to

$$
\dot{\hat{z}}(t)=\mathcal{P}\left[\dot{\hat{z}}_{\text {pre }}(t)\right]=\dot{\hat{z}}_{\text {pre }}(t)-\mathcal{I}(\hat{z}) \frac{\hat{z}(t) \hat{z}(t)^{\top}}{r^{2} \bar{z}^{2}} \dot{\hat{z}}_{\text {pre }}(t)
$$

where $\mathcal{P}$ denotes the projection operator and the function $\mathcal{I}(\hat{z})$ is given by

$$
\mathcal{I}(\hat{z})=\left\{\begin{array}{l}
1,|\hat{z}(t)|=r^{2} \bar{z} \quad \text { and } \quad \hat{z}(t)^{\top} \dot{\hat{z}}_{\text {pre }}(t)>0 \\
0, \text { otherwise. }
\end{array}\right.
$$

By defining the matrix $S(\hat{z}) \triangleq \mathcal{I}(\hat{z}) \frac{\hat{z}(t) \hat{z}(t)^{\top}}{r^{2} \bar{z}^{2}} \in \mathbb{R}^{2 n+1}$, the constant vector $\delta_{z}^{\top} \triangleq C A=r^{2}\left[\begin{array}{lllllll}0 & \alpha_{1} & 0 & \alpha_{2} & \cdots & 0 & \alpha_{n}\end{array}\right] \in$ $\mathbb{R}^{2 n+1}$, the time-varying vector

$$
\hat{\Phi}(t) \triangleq \frac{\hat{\Omega}(t)}{r^{2}}-r^{2} \Lambda
$$

depending upon the adapted parameter vector $\theta(t)$ through $\hat{\Omega}(t) \triangleq \theta(t)+r^{2} \mu \gamma(\hat{z}) y(t)$, and the vector function $\gamma(z)^{\top} \triangleq C \Gamma(z)=\left[\begin{array}{llll}z_{2} & z_{4} & \cdots & z_{2 n}\end{array}\right]$, we propose the following adaptive observer scheme:

$$
\left\{\begin{array}{l}
\dot{\hat{z}}(t)=A \hat{z}(t)+\Gamma(\hat{z}) \hat{\Phi}(t)+L(y(t)-C \hat{z}(t))-S(\hat{z}) \dot{\hat{z}}_{\text {pre }}(t) \\
\dot{\theta}(t)=F_{u}(t)+F_{c}(t)
\end{array}\right.
$$

where the two terms $F_{u}(t)$ and $F_{c}(t)$ forming the parameter-adaptation law are defined as follows

$$
\begin{aligned}
& F_{u}(t) \triangleq-r^{2} \mu \gamma(\hat{z})\left(\gamma(\hat{z})^{\top} \hat{\Phi}(t)+\delta_{z}^{\top} \hat{z}(t)\right)-r^{2} \mu \gamma(\dot{\hat{z}}) y(t) \\
& F_{c}(t) \triangleq-\Psi(t) \bar{S} \frac{\hat{\Omega}(t)}{\sqrt{n} \bar{\Omega}}
\end{aligned}
$$

with $\mu>0$ a user-defined scalar gain that tunes the convergence speed. $F_{u}(t)$ represents the unconstrained derivative of $\theta$, while $F_{c}(t)$ is a forcing term in charge 
of constraining the estimated parameters in the feasible set $\mathcal{W} . \Psi(t)$ in (10) is a binary switching signal that enables/disables the constraint-enforcing term $F_{c}(t)$ :

$$
\Psi(t)=\left\{\begin{array}{l}
1,|\hat{\Omega}(t)|=\sqrt{n} \bar{\Omega} \\
0,|\hat{\Omega}(t)|<\sqrt{n} \bar{\Omega}
\end{array}\right.
$$

Moreover, $\bar{S} \in \mathbb{R}_{>0}$ is a user-defined constant to be chosen large enough such that

$$
\bar{S} \geq \max _{\substack{(\hat{z}, z) \in \mathcal{Z}^{2} \\ \hat{\Omega} \in \mathcal{W}}}\left\{\left|\frac{d}{d t}\left(r^{2} \mu \gamma(\hat{z}) y(t)\right)+F_{u}(t)\right|\right\} .
$$

The upcoming analysis is carried out in order to show the existence of a finite $\bar{S}$ satisfying (12). When the forcing term $F_{c}(t)$ is non-null it holds that

$$
\Psi(t)=1 \text { and }|\hat{\Omega}(t)|=\sqrt{n} \bar{\Omega} .
$$

We can expand the right hand side of (12) by

$$
\begin{gathered}
\frac{d}{d t}\left(r^{2} \mu \gamma(\hat{z}) y(t)\right)+F_{u}(t)=r^{2} \mu \gamma(\dot{\hat{z}}) y(t)+r^{2} \mu \gamma(\hat{z}) \dot{y}(t) \\
-r^{2} \mu \gamma(\hat{z})\left(\gamma(\hat{z})^{\top} \hat{\Phi}(t)+\delta_{z}^{\top} \hat{z}(t)\right)-r^{2} \mu \gamma(\dot{\hat{z}}) y(t) \\
=r^{2} \mu \gamma(\hat{z})\left(\sum_{i=1}^{n} \omega_{i}^{2} z_{2 i}(t)+\dot{d}(t)\right) \\
-r^{2} \mu \gamma(\hat{z})\left(\gamma(\hat{z})^{\top} \hat{\Phi}(t)+\delta_{z}^{\top} \hat{z}(t)\right),
\end{gathered}
$$

the boundedness of which is implied by the boundedness of $z(t), \hat{z}(t), \Omega$ and $\dot{d}(t)$. Therefore there always exists a sufficient large $\bar{S}$, such that (12) is verified. In view of (10) and (13), it is immediate to show that

$$
\dot{\hat{\Phi}}=\frac{1}{r^{2}} \dot{\hat{\Omega}}=\frac{1}{r^{2}}\left(\frac{d}{d t}\left(r^{2} \mu \gamma(\hat{z}) y(t)\right)+F_{u}(t)-\bar{S} \frac{\hat{\Omega}(t)}{|\hat{\Omega}(t)|}\right) .
$$

Hence, in virtue of (12) we have that $\hat{\Omega}^{\top} \dot{\hat{\Omega}} \leq$ 0 , with $|\hat{\Omega}(t)|=\sqrt{n} \bar{\Omega}$ which indicates that the forcing term is strong enough to steer the trajectory of the spectral parameter estimates back into the feasible domain.

Given the estimates $\hat{\Omega}(t)$ and $\hat{z}(t)$, the frequency, amplitude and phase estimates for the $i$-th sinusoidal component can be retrieved by:

$$
\begin{aligned}
& \hat{\omega}_{i}(t)=\sqrt{\hat{\Omega}_{i}(t)}, \hat{a}_{i}(t)=\sqrt{\hat{z}_{2 i-1}^{2}(t)+\frac{\hat{\omega}_{i}^{2}(t)}{r^{4}} \hat{z}_{2 i}^{2}(t)}, \\
& \hat{\varphi}_{i}(t)=\angle\left(\frac{\hat{\omega}_{i}(t)}{r^{2}} \hat{z}_{2 i}(t)+j \hat{z}_{2 i-1}(t)\right), i=1,2 \ldots, n,
\end{aligned}
$$

and the offset is directly estimated by: $\hat{a}_{0}(t)=\hat{z}_{2 n+1}(t)$.

\section{Stability Analysis}

In this section, we address the stability of the adaptive observer. The following assumption is needed in order to prove the convergence of the proposed adaptive observer.

Assumption 4 (PE) The signal vector $\gamma(z)$ is persistently exciting ${ }^{1}$, in $\mathbb{R}^{n}$ with a level of excitation $\epsilon>0$, i.e. $\exists T_{0}>0$ such that

$$
\frac{1}{T_{0}} \int_{t}^{t+T_{0}} \gamma(z(\tau)) \gamma(z(\tau))^{\top} d \tau \geq \epsilon I, \forall t \geq 0 .
$$

Next, let us introduce a few instrumental error variables: $\tilde{z}(t) \triangleq \hat{z}(t)-z(t), \tilde{\Phi}(t) \triangleq \hat{\Phi}(t)-\Phi$ and $\tilde{\Omega}(t) \triangleq \hat{\Omega}(t)-\Omega$. Due to (5), it holds that $\tilde{\Phi}(t)=\frac{1}{r^{2}} \tilde{\Omega}(t)$. The dynamics of the state estimation error $\tilde{z}(t)$ can be written as

$$
\begin{aligned}
\dot{\tilde{z}}(t)= & (A-L C) \tilde{z}(t)+\Gamma(\hat{z}) \tilde{\Phi}+M(\Omega) \tilde{z} \\
& -S(\hat{z}) \dot{\hat{z}}_{\text {pre }}(t)+L d(t)
\end{aligned}
$$

where we use the relationship $\Gamma(\tilde{z}) \Phi=M(\Omega) \tilde{z}$, owing to the linearity of the map $\Gamma(\cdot)$ and with the sparse matrix $M(\Omega) \in \mathbb{R}^{(2 n+1) \times(2 n+1)}$ whose non-zero elements are given by $M_{2 i-1,2 i}(\Omega)=\omega_{i}^{2} / r^{2}-r^{2} \alpha_{i}, \forall i=1,2, \cdots, n$. Some intermediate results concerning the Lyapunov stability characterization of the above dynamics will be given in the following. It is worth noting that the matrix $A-L C+q I$ is Hurwitz for any $q \in \mathbb{R}_{>0}$ such that:

$$
q<\underline{g} \triangleq \min _{i \in\{1, \cdots, 2 n+1\}}\left\{-g_{i}\right\}
$$

where $g_{i} \in \mathbb{R}_{<0}, i=1,2, \cdots, 2 n+1$ are the eigenvalues of the dynamic matrix $A-L C$. Therefore, there always exists a positive symmetric matrix $T \in \mathbb{R}^{(2 n+1) \times(2 n+1)}$ that solves the following Lyapunov equation:

$$
(A-L C+q I)^{\top} T+T(A-L C+q I)=-q I .
$$

for any $q \in \mathbb{R}_{>0}$ verifies (15). Consider a positive symmetric matrix $P \in \mathbb{R}^{(2 n+1) \times(2 n+1)}$ such that $P^{2}=T$. It is immediate to show that $P(A-L C) P^{-1}$ is Hurwitz, thus there exists a positive-definite symmetric matrix $P_{s} \in \mathbb{R}^{(2 n+1) \times(2 n+1)}$ that solves the next Lyapunov equation:

$$
\left(P(A-L C) P^{-1}\right)^{\top} P_{s}+P_{s}\left(P(A-L C) P^{-1}\right)=-2 q_{s} I,
$$

\footnotetext{
${ }^{1}$ Persistent excitation assumption is a quite standard and not restrictive assumption in the framework of the parameter estimation of sinusoids $[4,15,20]$.
} 
for any $q_{s} \in \mathbb{R}_{>0}$. In order to derive an upper bound for $\overline{p_{s}} \triangleq \max \operatorname{eig}\left(P_{s}\right)$, we resort now to the following technical result of [32]: let $X$ be a Hurwitz matrix such that $X_{s}=\left(X+X^{\top}\right) / 2<0$; then the maximum solution of the Lyapunov equation $X^{\top} P_{x}+P_{x} X=-Q$, for any $Q>0$ such that $Q X_{s}^{-1}<0$ verifies the upper bound: $\max \operatorname{eig}\left(P_{x}\right) \leq \max \operatorname{eig}\left(-Q X_{s}\right)^{-1}$. This result applies to our formulation letting $X=P(A-L C) P^{-1}, P_{x}=P_{s}$ and $Q=2 q_{s} I$. Indeed, it can be readily verified (rightand-left-multiplying both sides of (16) by $P^{-1}$ ) that

$$
\begin{array}{r}
X_{s}=\frac{1}{2}\left(P^{-1}(A-L C)^{\top} P+P^{-1}(A-L C)^{\top} P\right) \\
=-2 q I-q P^{-2}
\end{array}
$$

is negative definite. Therefore, it holds that

$$
\overline{p_{s}} \leq q_{s} \max \operatorname{eig}\left(-X_{s}^{-1}\right)=q_{s} \frac{1}{\min \operatorname{eig}\left(-X_{s}\right)}<\frac{q_{s}}{q} .
$$

The main result is stated in the following theorem.

Theorem 4.1 Given the perturbed multi-sinusoidal signal $y(t)$, if Assumptions 1-4 hold and the observer gain $L$ is designed such that the eigenvalues of $A-L C$ verify the following inequality:

$$
\underline{g}>q>\max \left\{40 n \bar{\Omega}^{2} \frac{\bar{z}^{2}}{\epsilon} \frac{\bar{p}^{2}}{\underline{p}^{2}}, 2\left(\frac{\bar{\Omega}}{r^{2}}+r^{2} \bar{\alpha}\right)+1\right\},
$$

where $\bar{p} \triangleq \max \operatorname{eig}(P), \underline{p} \triangleq \min \operatorname{eig}(P)$. Then the estimation error $\tilde{z}(t)$ and $\tilde{\Phi}(t)$ exponentially converge to a residual set

$$
\mathcal{D}=\left\{\tilde{z}, \tilde{\Phi}|| \tilde{z}|+| \tilde{\Phi} \mid \leq \sigma\left(\bar{d}_{1}, \bar{d}_{2}\right)\right\}
$$

where $\sigma\left(\bar{d}_{1}, \bar{d}_{2}\right) \triangleq \frac{2 e^{\kappa T_{0}}}{\kappa \min \left\{\underline{p_{s}} \underline{p}^{2}, 1\right\}}\left(\mu \bar{d}_{2}^{2}+\frac{q_{s}}{q^{2}} \bar{p}^{2}|L|^{2} \bar{d}_{1}^{2}\right)$ is a positive function with respect to $\bar{d}_{1}, \bar{d}_{2}$.

Proof. Consider the following candidate Lyapunov function that accounts for $\tilde{z}(t)$ and $\tilde{\Phi}(t)$ :

$$
V(\tilde{z}(t), \tilde{\Phi}(t))=\frac{1}{2}\left(\tilde{z}(t)^{\top} P P_{s} P \tilde{z}(t)+\tilde{\Phi}(t)^{\top} \tilde{\Phi}(t)\right),
$$

where the positive symmetric matrices $P$ and $P_{s}$ are defined in the equation (16) and (17), respectively. Introducing the auxiliary variable $\tilde{x}(t)=P \tilde{z}(t) \in \mathbb{R}^{2 n+1}$ and resorting to (14), the dynamics of $\tilde{x}(t)$ write:

$$
\begin{aligned}
\dot{\tilde{x}}(t)= & P(A-L C) P^{-1} \tilde{x}(t)+P(\Gamma(\hat{z}) \tilde{\Phi}+M(\Omega) \tilde{z}(t)) \\
& -P\left(S(\hat{z}) \dot{\hat{z}}_{\text {pre }}(t)-L d(t)\right) .
\end{aligned}
$$

In view of $(8)$ and $(9), \tilde{\Phi}(t)$ evolves according to

$$
\begin{aligned}
\dot{\tilde{\Phi}}(t)= & -\mu \gamma(\hat{z}) \gamma(\hat{z})^{\top} \tilde{\Phi}(t)-\mu \gamma(\hat{z}) \gamma(\tilde{z})^{\top} \Phi(t)+\mu \gamma(\hat{z}) \dot{d}(t) \\
& -\mu \gamma(\hat{z}) \delta_{z}^{\top} \tilde{z}(t)+\frac{F_{c}(t)}{r^{2}} .
\end{aligned}
$$

In the sequel, we drop the dependence of time-varying variables (e.g., $\left.\dot{\tilde{z}}(t), \dot{\hat{z}}_{\text {pre }}(t), \dot{\tilde{x}}(t), d(t)\right)$ on $t$ for the sake of brevity. Then, using the auxiliary variables $\tilde{x}$, Lyapunov function can be written as $V(\tilde{z}, \tilde{\Phi})=\frac{1}{2}\left(\tilde{x}^{\top} P_{s} \tilde{x}+\tilde{\Phi}^{\top} \tilde{\Phi}\right)$. By means of (17), (22) and(23), $\dot{V}(\tilde{z}, \tilde{\Phi})$ becomes

$$
\begin{gathered}
\dot{V}(\tilde{z}, \tilde{\Phi}) \leq-q_{s} \tilde{x}^{\top} \tilde{x}+\tilde{x}^{\top} P_{s} P(\Gamma(\hat{z}) \tilde{\Phi}+M(\Omega) \tilde{z}) \\
+\tilde{x}^{\top} P_{s} P L d-\mu \tilde{\Phi}^{\top} \gamma(\hat{z}) \gamma(\hat{z})^{\top} \tilde{\Phi} \\
-\mu \tilde{\Phi}^{\top} \gamma(\hat{z})\left(\delta_{z}^{\top} \tilde{z}+\gamma(\tilde{z})^{\top} \Phi\right)+\mu \tilde{\Phi}^{\top} \gamma(\hat{z}) \dot{d}
\end{gathered}
$$

where we have taken advantage of the fact that the term $-\tilde{x}^{\top} P_{s} P S(\hat{z}) \dot{\hat{z}}_{\text {pre }}(t)$ and $\tilde{\Phi}^{\top} F_{c}(t)$ are non-positive, which are the results of the the projection operation in $(7)$ and constrain term $F_{c}(t)$ in $(10)$, respectively. By applying the Young's inequality [13], (19) and using the relation that $\Gamma(\hat{z})^{\top} \Gamma(\hat{z}) \leq r^{4} \bar{z}^{2} I, \forall \hat{z}(t) \in \mathbb{R}^{2 n+1}$, we get:

$$
\begin{gathered}
\tilde{x}^{\top} P_{s} P \Gamma(\hat{z}) \tilde{\Phi} \leq \frac{q_{s}}{4} \tilde{x}^{\top} \tilde{x}+\frac{q_{s}}{q^{2}} r^{4} \bar{z}^{2} \tilde{\Phi}^{\top} P^{2} \tilde{\Phi} \\
\tilde{x}^{\top} P_{s} P M(\Omega) \tilde{z} \leq \frac{q_{s}}{q}\|M(\Omega)\| \tilde{x}^{\top} \tilde{x} .
\end{gathered}
$$

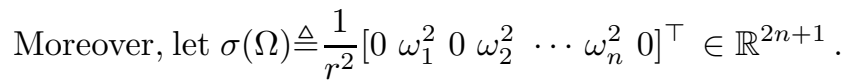
It holds that $\delta_{z}^{\top} \tilde{z}+\gamma(\tilde{z})^{\top} \Phi=\sigma(\Omega) \tilde{z}$, hence,

$$
\begin{array}{r}
-\mu \tilde{\Phi}^{\top} \gamma(\hat{z})\left(\delta_{z}^{\top} \tilde{z}+\gamma(\tilde{z})^{\top} \Phi\right)=-\mu \tilde{\Phi}^{\top} \gamma(\hat{z}) \sigma(\Omega) \tilde{z} \\
\leq \frac{\mu}{2} \tilde{\Phi}^{\top} \gamma(\hat{z}) \gamma(\hat{z})^{\top} \tilde{\Phi}+\frac{\mu}{2}|\sigma(\Omega)|^{2} \tilde{z}^{\top} \tilde{z}
\end{array}
$$

Thanks to Assumption 2, the upper bounds of $\tilde{x}^{\top} P_{s} P L d$ and $\mu \tilde{\Phi}^{\top} \gamma(\hat{z}) \dot{d}$ are established:

$$
\begin{gathered}
\tilde{x}^{\top} P_{s} P L d \leq \frac{q_{s}}{4} \tilde{x}^{\top} \tilde{x}+\frac{q_{s}}{q^{2}}|P L|^{2} \bar{d}_{1}^{2} \\
\mu \tilde{\Phi}^{\top} \gamma(\hat{z}) \dot{d} \leq \frac{\mu}{4} \tilde{\Phi}^{\top} \gamma(\hat{z}) \gamma(\hat{z})^{\top} \tilde{\Phi}+\mu \bar{d}_{2}^{2} .
\end{gathered}
$$

Applying (25)-(29) to (24), we can obtain

$$
\begin{aligned}
& \dot{V}(\tilde{z}, \tilde{\Phi}) \leq-\frac{\mu}{4} \tilde{\Phi}^{\top} \gamma(\hat{z}) \gamma(\hat{z})^{\top} \tilde{\Phi}+\frac{q_{s}}{q^{2}} r^{4} \bar{z}^{2} \bar{p}^{2} \tilde{\Phi}^{\top} \tilde{\Phi} \\
& -\tilde{z}^{\top}\left(\left(\frac{q_{s}}{2}-\frac{q_{s}}{q} \| M(\Omega)||\right) \underline{p}^{2}-\frac{\mu}{2}|\sigma(\Omega)|^{2}\right) \tilde{z}+\bar{d} .
\end{aligned}
$$

where $\bar{p}=\max \operatorname{eig}(P), \underline{p}=\min \operatorname{eig}(P)$ and $\bar{d}=\mu \bar{d}_{2}^{2}+$ 
$\frac{q_{s}}{q^{2}} \bar{p}^{2}|L|^{2} \bar{d}_{1}^{2}$. The linearity of the map $\gamma(\cdot)$ implies that

$\gamma(\hat{z}) \gamma(\hat{z})^{\top}=\gamma(z+\tilde{z}) \gamma(z+\tilde{z})^{\top} \geq \gamma(z) \gamma(z)^{\top}+2 \gamma(z) \gamma(\tilde{z})^{\top}$

by which we derive an upper bound of the first term on the right hand side of (30) as follows

$-\frac{\mu}{4} \tilde{\Phi}^{\top} \gamma(\hat{z}) \gamma(\hat{z})^{\top} \tilde{\Phi} \leq-\frac{\mu}{8} \tilde{\Phi}^{\top} \gamma(z) \gamma(z)^{\top} \tilde{\Phi}+\frac{2 \mu n \bar{\Omega}^{2}}{r^{4}} \tilde{z}^{\top} \tilde{z}$.

where we made use of the relations that $\tilde{\Phi}^{\top} \gamma(\tilde{z}) \gamma(\tilde{z})^{\top} \tilde{\Phi} \leq \tilde{z}^{\top}\left\|\tilde{\Phi} \tilde{\Phi}^{\top}\right\| \tilde{z}$ and $\left\|\tilde{\Phi} \tilde{\Phi}^{\top}\right\| \leq|\tilde{\Phi}|^{2}=$ $\frac{1}{r^{4}}|\tilde{\Omega}|^{2} \leq \frac{4 n \bar{\Omega}^{2}}{r^{4}}$. Under Assumption 1, the terms $|\sigma(\Omega)|^{2}$ and $|M(\Omega)|$ can be upper bounded by

$\max _{\Omega:|\Omega|_{\infty} \leq \bar{\Omega}}\left\{|\sigma(\Omega)|^{2}\right\} \leq \frac{n \bar{\Omega}^{2}}{r^{4}}, \max _{\Omega:|\Omega|_{\infty} \leq \bar{\Omega}}\{|M(\Omega)|\} \leq \frac{\bar{\Omega}}{r^{2}}+r^{2} \bar{\alpha}$.

Applying (31) and (32), we can finally get:

$$
\dot{V}(\tilde{z}, \tilde{\Phi}) \leq-\beta(\hat{z}, z) V(\tilde{z}, \tilde{\Phi})+\bar{d}
$$

with

$$
\begin{aligned}
& \beta(\hat{z}, z) \triangleq 2 \min \left\{\min \operatorname{eig}\left(\frac{\mu}{8} \gamma(z) \gamma(z)^{\top}-\frac{q_{s}}{q^{2}} r^{4} \bar{z}^{2} \bar{p}^{2} I\right),\right. \\
& \left.\frac{q}{q_{s} \bar{p}^{2}}\left(\left(\frac{q_{s}}{2}-\frac{q_{s}}{q}\left(\frac{\bar{\Omega}}{r^{2}}+r^{2} \bar{\alpha}\right)\right) \underline{p}^{2}-\frac{5 \mu n}{2 r^{4}} \bar{\Omega}^{2}\right)\right\} .
\end{aligned}
$$

According to [17, Chapter 8], the Lyapunov function $V$ is exponentially bounded if $\beta(\hat{z}, z)$ is persistently exciting with a level of excitation $\kappa>0$, i.e. $\exists T_{0}>0$, such that

$$
\frac{1}{T_{0}} \int_{t}^{t+T_{0}} \beta(\hat{z}, z) d \tau \geq \kappa>0, \forall t \geq 0 .
$$

Therefore, the next lines are devoted to show that $\beta$ verifies (35). From (20), it is immediate to obtain

$$
q>40 n \bar{\Omega}^{2} \frac{\bar{z}^{2}}{\epsilon} \frac{\bar{p}^{2}}{\underline{p}^{2}}, \quad q-2\left(\frac{\bar{\Omega}}{r^{2}}+r^{2} \bar{\alpha}\right)>1 .
$$

Multiply the above inequalities side-by-side to get:

$$
q\left(q-2\left(\frac{\bar{\Omega}}{r^{2}}+r^{2} \bar{\alpha}\right)\right)>40 n \bar{\Omega}^{2} \frac{\bar{z}^{2}}{\epsilon} \frac{\bar{p}^{2}}{\underline{p}^{2}} .
$$

Multiplying both sides of (36) by the positive tuning gain $\mu$ and $\frac{1}{r^{4}}$, the following inequality is verified

$$
\frac{5 \mu n \bar{\Omega}^{2}}{\left(1-\frac{2\left(\frac{\bar{\Omega}}{r^{2}}+r^{2} \bar{\alpha}\right)}{q}\right) \underline{p}^{2} r^{4}}<\frac{\mu q^{2} \epsilon}{8 r^{4} \bar{z}^{2} \bar{p}^{2}} .
$$

Being the Lyapunov parameter $q_{s}>0$ arbitrary, let us pick $q_{s}$ in the non-empty set bounded by extrema of the above inequality. Therefore $q_{s}$ verifies:

$$
\begin{aligned}
& q_{s}> \frac{5 \mu n \bar{\Omega}^{2}}{\left(1-\frac{2\left(\frac{\bar{\Omega}}{r^{2}}+r^{2} \bar{\alpha}\right)}{q}\right) \underline{p}^{2} r^{4}}, \\
& q_{s}<\frac{\mu q^{2} \epsilon}{8 r^{4} \bar{z}^{2} \bar{p}^{2}} .
\end{aligned}
$$

First, multiplying both sides of (37a) by $\left(1 / 2-\left(\bar{\Omega} / r^{2}+r^{2} \bar{\alpha}\right) / q\right) \underline{p}^{2}$, we get

$$
\left(\frac{q_{s}}{2}-\frac{q_{s}}{q}\left(\frac{\bar{\Omega}}{r^{2}}+r^{2} \bar{\alpha}\right)\right) \underline{p}^{2}>\frac{5 \mu n}{2 r^{4}} \bar{\Omega}^{2},
$$

On the other hand, in view of the PE Assumption 4 and inequality (37b), the integral of the first argument in (34) over the time interval $\left[t, t+T_{0}\right]$ can be lower bounded by

$$
\begin{aligned}
& \frac{1}{T_{0}} \int_{t}^{t+T_{0}} \frac{\mu}{8} \gamma(z) \gamma(z)^{\top}-\frac{q_{s}}{q^{2}} r^{4} \bar{z}^{2} \bar{p}^{2} I d \tau \\
\geq & \left(\frac{\mu}{8} \epsilon-\frac{q_{s}}{q^{2}} r^{4} \bar{z}^{2} \bar{p}^{2}\right) I=\frac{r^{4} \bar{z}^{2} \bar{p}^{2}}{q^{2}}\left(\frac{\mu q^{2} \epsilon}{8 r^{4} \bar{z}^{2} \bar{p}^{2}}-q_{s}\right) I>0,
\end{aligned}
$$

$\forall t>0$. Now, defining

$$
\begin{gathered}
\kappa_{1} \triangleq \frac{r^{4} \bar{z}^{2} \bar{p}^{2}}{q^{2}}\left(\frac{\mu q^{2} \epsilon}{8 r^{4} \bar{z}^{2} \bar{p}^{2}}-q_{s}\right) \\
\kappa_{2} \triangleq \frac{q}{q_{s} \bar{p}^{2}}\left(\left(\frac{q_{s}}{2}-\frac{q_{s}}{q}\left(\frac{\bar{\Omega}}{r^{2}}+r^{2} \bar{\alpha}\right)\right) \underline{p}^{2}-\frac{5 \mu n}{2 r^{4}} \bar{\Omega}^{2}\right),
\end{gathered}
$$

we can conclude that any $\kappa \in\left(0,2 \min \left\{\kappa_{1}, \kappa_{2}\right\}\right]$ verifies (35). For the sake of brevity, let us denote $V(\tilde{z}(t), \tilde{\Phi}(t))$ and $\beta(\hat{z}(t), z(t))$ by $V(t)$ and $\beta(t)$ respectively. By the Gronwall-Bellman Lemma, the value of the Lyapunov function (33) can be bounded as follows:

$$
\begin{aligned}
V(t) & \leq e^{-\int_{0}^{t} \beta(\tau) d \tau} V(0)+\int_{0}^{t} \bar{d} e^{-\int_{\tau}^{t} \beta(s) d s} d \tau \\
& \leq e^{-\int_{0}^{t} \beta(\tau) d \tau} V(0)+\bar{d} \int_{0}^{t} e^{-\kappa\left(t-\tau-T_{0}\right)} d \tau \\
& \leq e^{-\kappa t} V(0)+\frac{\bar{d} e^{\kappa T_{0}}}{\kappa}\left(1-e^{-\kappa t}\right)
\end{aligned}
$$

for $\forall t>T_{0}$. Therefore, we have

$$
\frac{\underline{p}_{s} \underline{p}^{2}}{2}|\tilde{z}(t)|+\frac{1}{2}|\tilde{\Phi}(t)| \leq V(t) \leq \frac{\bar{d} e^{\kappa T_{0}}}{\kappa}+\rho(t) .
$$


where $p_{s}=\min \operatorname{eig}\left(P_{s}\right)$ and $\rho(t)$ is an exponentially decaying term. Therefore, the total estimation errors $|\tilde{z}(t)|$ and $|\tilde{\Phi}(t)|$ exponentially converge to the residual set $\mathcal{D}$ (see $(21))$, thus concluding the proof.

\section{Parameters Tuning Guidelines}

The stability analysis also provides some tuning guidelines for the parameters of the proposed estimation algorithm, depending on the assumed disturbance level (see Assumption 2) and on the required asymptotic accuracy. The design procedure consists of two steps. First, set $r=1$ and choose $n$ arbitrary constant parameters $\Lambda=\left[\begin{array}{llll}\alpha_{1} & \alpha_{2} & \cdots & \alpha_{n}\end{array}\right]$, such that $\alpha_{i} \neq \alpha_{j}$ for $i \neq j, \forall i, j=1,2 \cdots, n$. In view of the inequality (20), choose the parameters $q \in \mathbb{R}_{>0}$ and $g_{i} \in \mathbb{R}_{<0}$ such that $\underline{g}>q>2\left(\frac{\bar{\Omega}}{r^{2}}+r^{2} \bar{\alpha}\right)+1$, where $\bar{\Omega}$ is defined in the Assumption 1 and $\bar{\alpha}$ in (3).

Then solve the equation (16) to obtain the condition number of the positive definite matrix $T$ (i.e., $\bar{p}^{2} / p^{2}$ ). Together with the prior knowledge of the upper bound $\bar{z}$, the minimum value of $q$ ensuring the stability of the estimator, denoted by $\underline{q}$, can be calculated by exploiting $(20)$ :

$$
\underline{q} \triangleq \max \left\{40 n \bar{\Omega}^{2} \frac{\bar{z}^{2}}{\epsilon} \frac{\bar{p}^{2}}{\underline{p}^{2}}, 2\left(\frac{\bar{\Omega}}{r^{2}}+r^{2} \bar{\alpha}\right)+1\right\} .
$$

If $q>\underline{q}$, the design of the adaptive observer is completed. If not, choose another constant $q^{\prime}$ such that $q^{\prime}>\underline{q}$. Then set $r^{\prime}=\sqrt{q^{\prime} / q} r$ and redesign the observer gain as $L^{\prime}=$ $q^{\prime} / q L$. It turns then out that $A\left(r^{\prime}\right)=q^{\prime} / q A(r)$ and the poles of the new observer are shifted to $g_{i}^{\prime}=q^{\prime} / q g_{i}, \forall i=$ $1,2, \cdots, 2 n+1$. We can immediately obtain that

$$
\underline{g}^{\prime}=\frac{q^{\prime}}{q} \underline{g}>\frac{q^{\prime}}{q} q=q^{\prime}
$$

Next, we show that the stability condition (20) is verified for the redesigned observer. Note that, the same matrix $T$ solving (16) also solves the Lyapunov equation for the redesigned observer:

$$
\left(\left(A\left(r^{\prime}\right)-L^{\prime} C\right)+q^{\prime} I\right)^{\top} T+T\left(\left(A\left(r^{\prime}\right)-L^{\prime} C\right)+q^{\prime} I\right)=-q^{\prime} I .
$$

Analogously, multiplying both sides of each term in (42) by $P^{-1}$, we obtain

$$
P^{-1}\left(A\left(r^{\prime}\right)-L^{\prime} C\right)^{\top} P+P\left(A\left(r^{\prime}\right)-L^{\prime} C\right) P^{-1}=-2 q^{\prime} I-q^{\prime} P^{-2} .
$$

Since $P^{-1}\left(A\left(r^{\prime}\right)-L^{\prime} C\right)^{\top} P$ is Hurwitz, the Lyapunov equation (17) and the inequality (19) is verified by $q^{\prime}$. Moreover, the condition number $\bar{p}^{2} / \underline{p}^{2}$ is invariant under the redesign, consequently, the first term in (40) is preserved, yielding to

$$
q^{\prime}>40 n \bar{\Omega}^{2} \frac{\bar{z}^{2}}{\epsilon} \frac{\bar{p}^{2}}{\underline{p}^{2}} .
$$

Additionally, since $q>2\left(\frac{\bar{\Omega}}{r^{2}}+r^{2} \bar{\alpha}\right)+1$ and $\frac{q^{\prime}}{q}>1$, the following inequality holds for the redesigned observer:

$$
q^{\prime}=\frac{q^{\prime}}{q} q>\frac{q^{\prime}}{q}\left(2\left(\frac{\bar{\Omega}}{r^{2}}+r^{2} \bar{\alpha}\right)+1\right)>2\left(\frac{\bar{\Omega}}{r^{\prime 2}}+r^{\prime 2} \bar{\alpha}\right)+1
$$

In view of the inequalities (41), (43) and (44), the stability condition in (20) is satisfied for the redesigned observer, thus completing the design.

\section{Simulation Results}

In this section, we provide some numerical examples to show the effectiveness of the proposed method. The algorithms taken for comparison are discretized by the $4^{\text {th }}$-order Runge-Kutta method with sampling period $T_{s}=1 \times 10^{-4} \mathrm{~s}$.

\section{Example 1}

In this example, we compare the proposed algorithm with a recent multi-FLL approach [10], the minimal dimension observer presented in [4] and the DREM method in [29]. The input signal is composed by two sinusoids: $y(t)=\sin (2 t)+\sin (5 t)$.

For the sake of fair comparison, all the methods are initialized with the same initial condition $\hat{\omega}(0)=[3,4]$ and are tuned to ensure similar convergence speed in the absence of disturbance. More specifically, the method in [4] is tuned with: $K=\left[k_{1}, k_{2}, k_{3}\right]=[1.2,0.47,0.06]$ and $\gamma_{1}=1$ and $\gamma_{2}=400$, while the method reported in [10] is set as $K_{s}=1.2, \gamma_{s 1}=1, \gamma_{s 2}=0.2$, and the tuning parameters of the method in [29] are set as $\lambda=1, \gamma_{1}=1, \gamma_{2}=5, k_{1}=k_{2}=50$. The parameters of the proposed estimator are chosen as: $\Lambda=[65,15]$, $\bar{g}=4, r=1$ and $\mu=5$. The behavior of the estimators is shown in Fig. 1. As it can be seen, all the methods succeed in detecting the frequencies with similar transient time. Next, the four algorithms are compared in presence of a disturbance $d(t)=0.25 \sin (10 t)$, which is added to the measurement: $\hat{y}(t)=\sin (2 t)+\sin (5 t)+d(t)$. As it can be noticed from Fig. 2, all the methods are capable to track both the frequencies in this scenario. However, the algorithm presented in [4] is more susceptible to high frequency disturbances than the other three methods. Example 2

In order to gain more insight into the features of the proposed adaptive observer, let us consider a multisinusoidal signal whose components exhibit a sudden (step-wise) frequency variation: $\hat{y}(t)=\sin (\omega(t) t)+$ $\sin (5 t)$, where $\omega(t)=2$, for $t \in[0,15)$ and $\omega(t)=4.5$, for $t \in[150,+\infty)$. 


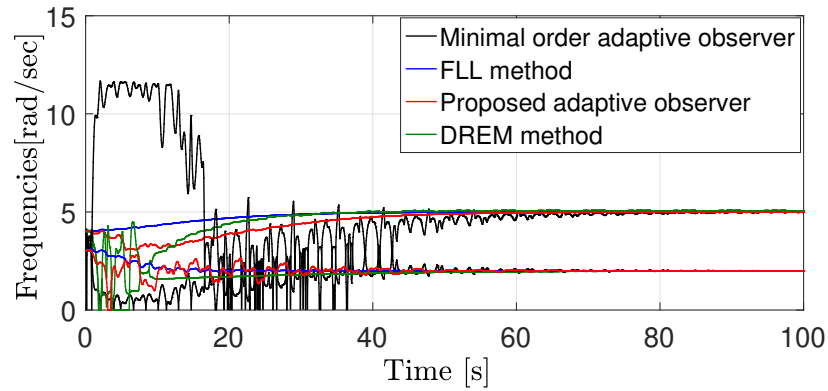

Fig. 1. Time behaviour of the estimated frequencies for an unperturbed multi-sinusoidal signal.

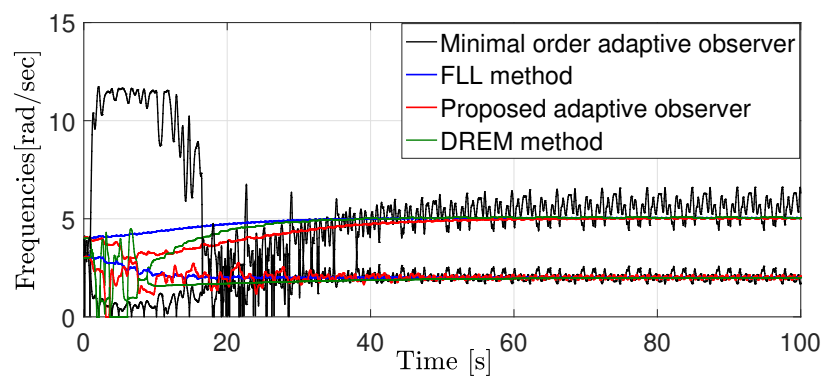

Fig. 2. Time behaviour of the estimated frequencies for a perturbed multi-sinusoidal signal.

At this stage, we compare the proposed method with the FLL and DREM approaches. All the algorithms are tuned to have a similar initial transient response. The results are reported in Fig. 3. As it can be noticed, the FLL [10] method struggles to identify the nearby frequencies after the step change, whilst the present algorithm and the DREM method favorably deal with the frequency change and lead to comparable stationary behaviour. Nevertheless, the proposed method has the advantage to provide direct estimates for the frequencies.

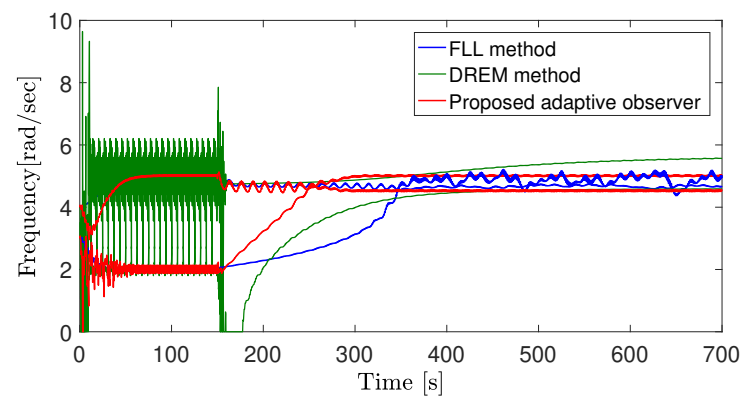

Fig. 3. Time behaviour of the estimated frequencies for an unperturbed multi-sinusoidal signal with a step frequency change at 150 s.

\section{Example 3}

Now, the present algorithm is simulated in a more challenging scenario, where the input consists of three sinusoidal signals and an unknown bias: $y(t)=2 \sin (2 t)+$ $4 \sin (5 t)+3 \sin (3 t)+0.5$. The parameters are set to:
$\Lambda=[250,65,15], r=1, \bar{g}=5$, while the adaptation gain $\mu$ is chosen as 0.05 , with initial condition $\hat{\omega}(0)=[1.5,2.5,6]$. Figures 4,5 and 6 show respectively the estimated frequencies, amplitudes (including the offset) and the phase estimation errors, which verify the effectiveness of the proposed methodology in this case.

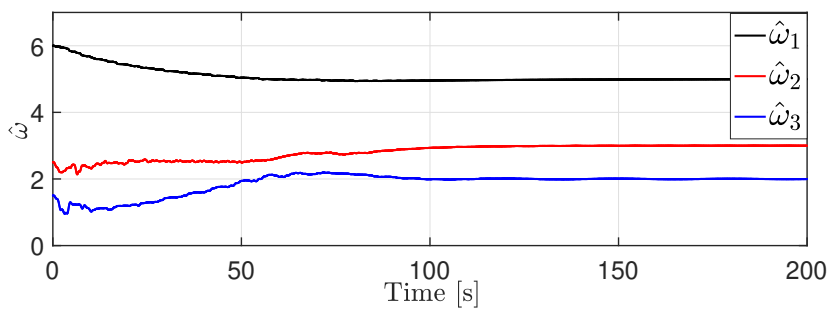

Fig. 4. Time behaviour of the estimated frequencies for a biased multi-sinusoidal signal.

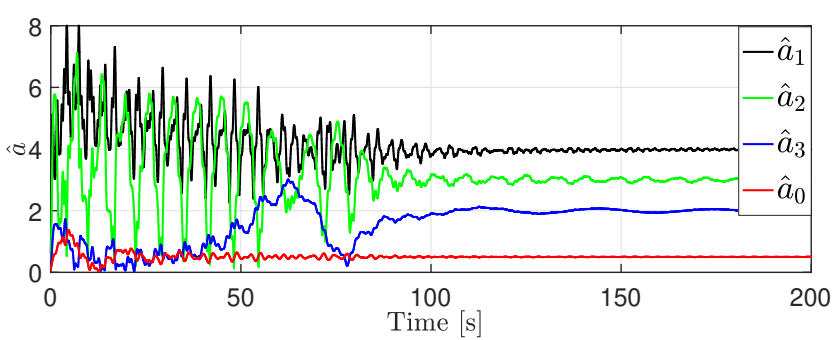

Fig. 5. Time behaviour of the estimated amplitudes and offset for a biased multi-sinusoidal signal.

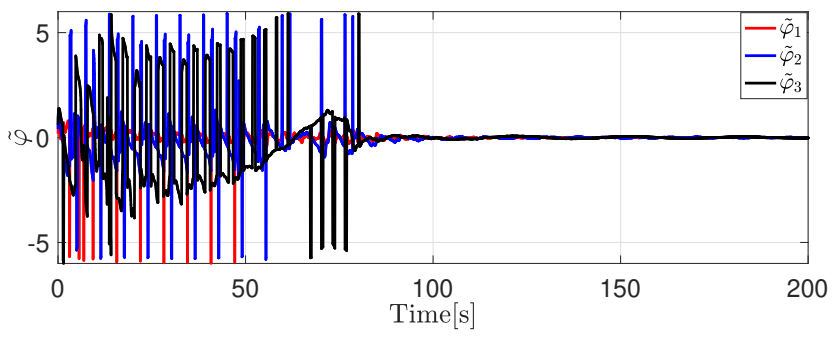

Fig. 6. Time behaviour of the phase estimation errors for a biased multi-sinusoidal signal.

\section{Concluding remarks}

In this paper, a novel adaptive observer-based estimator is proposed to address the AFP problem for a biased and perturbed multi-sinusoidal signal. The presented methodology allows for the direct adaptation of the squared-frequencies of the components instead of estimating the characteristic polynomial's coefficients of the signal-generator system. Moreover, the proposed estimator is characterized by a low dynamic order equal to $3 n$ or $3 n+1$ when featuring bias elimination. The effectiveness of the proposed algorithm has been verified by several comparative simulation examples. Future research efforts will be devoted to the development of realtime experimental results. 


\section{References}

[1] S. V. Aranovskiy, A. A. Bobtsov, R. Ortega, and A. A. Pyrkin. Improved transients in multiple frequencies estimation via dynamic regressor extension and mixing. IFAC-PapersOnLine, 49(13):99-104, 2016.

[2] S. Bittanti and S. M. Savaresi. On the parametrization and design of an extended kalman filter frequency tracker. IEEE Trans. on Automatic Control, 45(9):1718-1724, 2000.

[3] M. Bodson and G. Fedele. Editorial for the special issue on recent advances in adaptivemethods for frequency estimation with applications. International Journal of Adaptive Control and Signal Processing, 30:1547-1549, 2016.

[4] D. Carnevale and A.Astolfi. A minimal dimension observer for global frequency estimation. In Proc. IEEE American Control Conference, pages 5269-5274, 2008.

[5] D. Carnevale and A. Astolfi. A hybrid observer for frequency estimation of saturated multi-frequency signals. In IEEE Conference on Decision and Control and European Control Conference, pages 2577-2582, 2011.

[6] D. Carnevale, S. Galeani, M. Sassano, and A. Astolfi. Robust hybrid estimation and rejection of multi-frequency signals. International Journal of Adaptive Control and Signal Processing, pages 1649-1673, 2016.

[7] B. Chen, G. Pin, W. M. Ng, C. K. Lee, S. Y. R. Hui, and T. Parisini. An adaptive observer-based switched methodology for the identification of a perturbed sinusoidal signal: Theory and experiments. IEEE Trans. on Signal Processing, 62(24):6355-6365, 2014.

[8] Boli Chen, Gilberto Pin, and Thomas Parisini. An adaptive observer-based estimator for multi-sinusoidal signals. In Proc. IEEE American Control Conference, pages 2505-2510, 2014.

[9] G. Fedele and A. Ferrise. Non adaptive second order generalized integrator for identification of a biased sinusoidal signal. IEEE Trans. on Automatic Control, 57(7):1838-1842, 2012.

[10] G. Fedele and A. Ferrise. A frequency-locked-loop filter for biased multi-sinusoidal estimation. IEEE Trans. on Signal Processing, 62(5):1125-1134, 2014.

[11] G. Fedele, A. Ferrise, and P. Muraca. An adaptive quasinotch filter for a biased sinusoidal signal estimation. In 9th IEEE International Conference on Control and Automation, pages 1060-1065, 2011.

[12] X. Guo and M. Bodson. Analysis and implementation of an adaptive algorithm for the rejection of multiple sinusoidal disturbances. IEEE Trans. on Control Systems Technology, 17(1):40-50, 2009.

[13] G.H. Hardy, J.E. Littlewood, and G. Pólya. Inequalities. Cambridge Mathematical Library. Cambridge University Press, 1952.

[14] M. Hou. Estimation of sinusoidal frequencies and amplitudes using adaptive identifier and observer. IEEE Trans. on Automatic Control, 52(3):493-499, 2007.

[15] M. Hou. Parameter identification of sinusoids. IEEE Trans. on Automatic Control, 57(2):467-472, 2012.

[16] L. Hsu, R. Ortega, and G. Damm. A globally convergent frequency estimator. IEEE Trans. on Automatic Control, 44(4):698-713, 1999.

[17] P.A. Ioannou and J. Sun. Robust Adaptive Control. Control theory. PTR Prentice-Hall, 1996.

[18] M. Karimi-Ghartemani, S. Ali Khajehoddin, Praveen K. Jain, Alireza Bakhshai, and Mohsen Mojiri. Addressing DC component in PLL and notch filter algorithms. IEEE Trans. on Power Electronics, 27(1):78-86, 2012.

[19] M. Karimi-Ghartemani and A. K. Ziarani. A nonlinear time-frequency analysis method. IEEE Trans. on Signal Processing, 52(6):1585-1595, 2004.

[20] R. Marino and P.Tomei. Global estimation of $n$ unknown frequencies. IEEE Trans. on Automatic Control, 47(8):13241328, 2002 .

[21] M. Mojiri, M. Karimi-Ghartemani, and A. Bakhshai. Processing of harmonics and interharmonics using an adaptive notch filter. IEEE Trans. on Power Delivery, 25(2):534-542, 2010.

[22] Mohsen Mojiri and A. R. Bakhshai. Estimation of $n$ frequencies using adaptive notch filter. IEEE Trans. on Circuits and Systems-II, 54(4):338-342, 2007.

[23] G. Obregon-Pulido, B. Castillo-Toledo, and A. Loukianov. A globally convergent estimator for n-frequencies. IEEE Trans. on Automatic Control, 47(5):857-863, 2002.

[24] G. Pin, Y. Wang, B. Chen, and T. Parisini. Semi-global direct estimation of multiple frequencies with an adaptive observer having minimal parameterization. In IEEE Conference on Decision and Control (CDC), pages 3693-3698, 2015.

[25] Gilberto Pin, Boli Chen, Thomas Parisini, and Marc Bodson. Robust sinusoid identification with structured and unstructured measurement uncertainties. IEEE Trans. on Automatic Control, 2014.

[26] A. A. Pyrkin, A. A. Bobtsov, A. A. Vedyakov, and S. A.Kolyubin. Estimation of polyharmonic signal parameters. Automation and Remote Control, 76(8):14001416, 2015.

[27] B.F. Scala and R. Bitmead. Design of an extended kalman filter frequency tracker. IEEE Trans. on Signal Processing, 44(3):739-742, 1996.

[28] B. B. Sharma and I. N. Kar. Design of asymptotically convergent frequency estimator using contraction theory. IEEE Trans. on Automatic Control, 53(8):1932-1937, 2008.

[29] J. Wang, P. A. Gritsenko, S. V. Aranovskiy, A. A. Bobtsov, and A. A. Pyrkin. A method for increasing the rate of parametric convergence in the problem of identification of the sinusoidal signal parameters. Automation and Remote Control, 78(3):389-396, 2017.

[30] B. Wu and M.Bodson. A magnitude/phase-locked loop approach to parameter estimation of periodic signals. IEEE Trans. on Automatic Control, 48(4):612-618, 2003.

[31] X. Xia. Global frequency estimation using adaptive identifiers. IEEE Trans. on Automatic Control, 47(7):11881193, 2002

[32] K. Yasuda and K.Hirai. Upper and lower bounds on the solution of the algebraic matrix riccati and lyapunov equation. IEEE Trans. on Automatic Control, 24(6):483-487, 1979 . 